\title{
Scanning Tunneling Microscopy of Modulated Surface Structures
}

\author{
A. Prodan ${ }^{*}$, H. J. P. van Midden ${ }^{*}$, N. Jug ${ }^{*}$, F. W. Boswell ${ }^{* *}$, J. C. Bennett ${ }^{* * *}$, and H. Böhm ${ }^{* * * *}$ \\ *Institute Jožef Stefan, Jamova 39, SI-1000 Ljubljana, Slovenia \\ ** Department of Physics, University of Waterloo, Waterloo, Ontario, Canada N2L 3G1 \\ **** Department of Physics, Acadia University, Wolfville, Nova Scotia, Canada B0P 1X0 \\ ${ }^{* * * * *}$ Geosciences, Johannes Gutenberg University, D-55099 Mainz, Germany
}

Modulated structures are commonly found in transition-metal chalcogenides with compositions between $\mathrm{MX}_{4}$ and $\mathrm{M}_{3} \mathrm{X}_{4}$. Their origin is in the low-dimensional character of these compounds, which supports phenomena like charge density waves (CDW). These can be further stabilized at surfaces, where the bulk dimensionality is additionally reduced. Modern surface methods, combined with calculations of the electronic properties, can give a new insight into such phenomena.

Two weak incommensurate modes, superimposed onto the main breathing mode, were discovered during the initial TEM studies of $\mathrm{Nb}_{1-\mathrm{x}} \mathrm{Ta}_{\mathrm{x}} \mathrm{Te}_{4}$. They were detected at the $\mathrm{NbTe}_{4}$ side of the phase diagram as weak and diffuse satellites in overexposed electron diffraction patterns [1]. Although a bulk phenomenon, these modes were for the first time successfully resolved by STM [2]. This was possible due to the preferential cleavage of the $\mathrm{NbTe}_{4}$ crystals, which exposed complete tetragonal anti-prismatic columns. The weak transversal modes were observed as a surface phenomenon, superimposed onto the much stronger breathing mode (Fig.1).

The observed sliding of two apparently independent low-temperature CDWs in $\mathrm{NbSe}_{3}$ was recently explained on basis of a new model [3], which took into account statistically distributed nanodomains of both CDW modes. The results of earlier low-temperature STM [4] and satellite dark field TEM studies [5] were shown to be in a good accord with the model. It was further shown that sub-monolayer gold deposits on the $\mathrm{NbSe}_{3}$ van der Waals surface exhibit poorly correlated modulated regions, whose periodicities are comparable to those of the two CDWs in pure $\mathrm{NbSe}_{3}$. In another trichalcogenide, $\mathrm{ZrTe}_{3}$, a surface compositional modulation [6] was shown to be a result of ordered, surplus tellurium atoms, which occupied the interstitial positions on the (001) $\mathrm{ZrTe}_{3}$ surface. Calculations within the extended Hückel tight binding (EHTB) approximation show that these tellurium atoms also account for the relatively high metallic conductivity of this compound.

CDW domains of two orientational variants were detected at the edges of disk-like surface defects in $\mathrm{NbSe}_{2}$, which was an indication that the deformed regions of the $\mathrm{Se}-\mathrm{Nb}-\mathrm{Se}$ sandwiches assumed an octahedral rather than trigonal-prismatic coordination [7]. It was further shown that surface domain boundaries between orientational variants in the monoclinic $\mathrm{NbTe}_{2}$ were often strongly modulated (Fig.2) with periodicities identical to those of the adjacent domains [8]. In contrast to the modulation in $\mathrm{NbSe}_{2}$, the $\mathrm{NbTe}_{2}$ modulation was interpreted on basis of a misfit between the domain boundaries across the van der Waals gaps.

Finally, the $\mathrm{Nb}_{3} \mathrm{X}_{4}(\mathrm{X}=\mathrm{S}, \mathrm{Se}, \mathrm{Te})$ compounds, characterized by large hexagonal tunnels [9], were studied by means of TEM and STM. From the three compounds forming the family only $\mathrm{Nb}_{3} \mathrm{Te}_{4}$ shows bulk conductivity anomalies at $95 \mathrm{~K}$, which were attributed to a CDW with a modulation vector $\mathbf{q}= \pm\left(1 / 3 \mathbf{a}^{*}+1 / 3 \mathbf{b}^{*}+3 / 7 \mathbf{c}^{*}\right)$. Intercalation with indium and thallium raises the CDW onset 
temperature in $\mathrm{Nb}_{3} \mathrm{Te}_{4}$ and supports $\mathrm{CDW}$ formation in the other two compounds [10,11], without significantly deforming the host structure. The electronic properties, calculated within the EHTB method, confirmed that intercalation flattens the Fermi surfaces and consequently improves the onedimensional character of these compounds. Poorly correlated modulation along the hexagonal columns was consistently observed in room-temperature STM images of these compounds, indicating that precursor effects to full CDW formation are surface supported phenomena.

\section{References:}

[1] F. W. Boswell and A. Prodan, Phys. Rev. B 34 (1986) 2979.

[2] A. Prodan et al., Phys. Rev. B 57 (1998) 6235.

[3] A. Prodan et al., Phys. Rev. B 64 (2001) 115423-1.

[4] Z. Dai et al., Phys. Rev. Lett. 66 (1991) 1318.

[5] K. K. Fung and J. W. Steeds, Phys. Rev. Lett., 45 (1980) 1696.

[6] A. Prodan et al., Surf. Sci., $482-5$ (2001), 1368.

[7] N. Ramšak et al., Phys. Rev. B 60 (1999), 4513.

[8] D. Cukjati et al., to appear in J. Cryst. Growth (2002).

[9] K. Selte and A. Kjekshus, Acta Cryst. 17 (1964) 1568.

[10] F. W. Boswell and J. C. Bennett, Mat. Res. Bull. 31 (1996) 1083.

[11] F. W. Boswell et al., J. Sol. St. Chem. 144 (1999) 454.

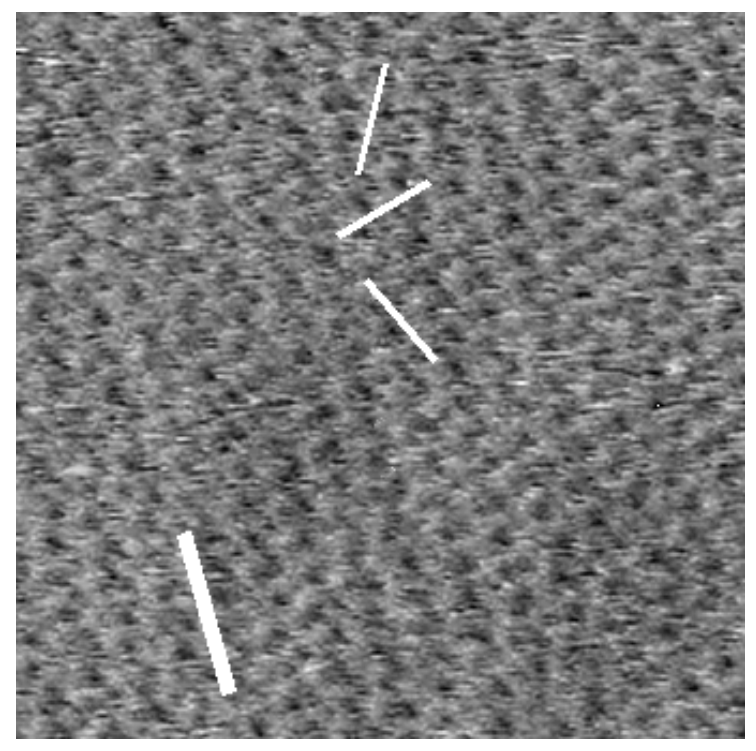

FIG.1. STM image of a $\{100\}$ surface of $\mathrm{NbTe}_{4}$, with the colums (thick) and the CDW modes (thin) indicated; $(10 \mathrm{~nm})^{2}$ area, constant height mode, $\mathrm{U}_{\mathrm{g}}=1 \mathrm{mV}, \mathrm{I}_{\mathrm{t}}=2.5 \mathrm{nA}$.

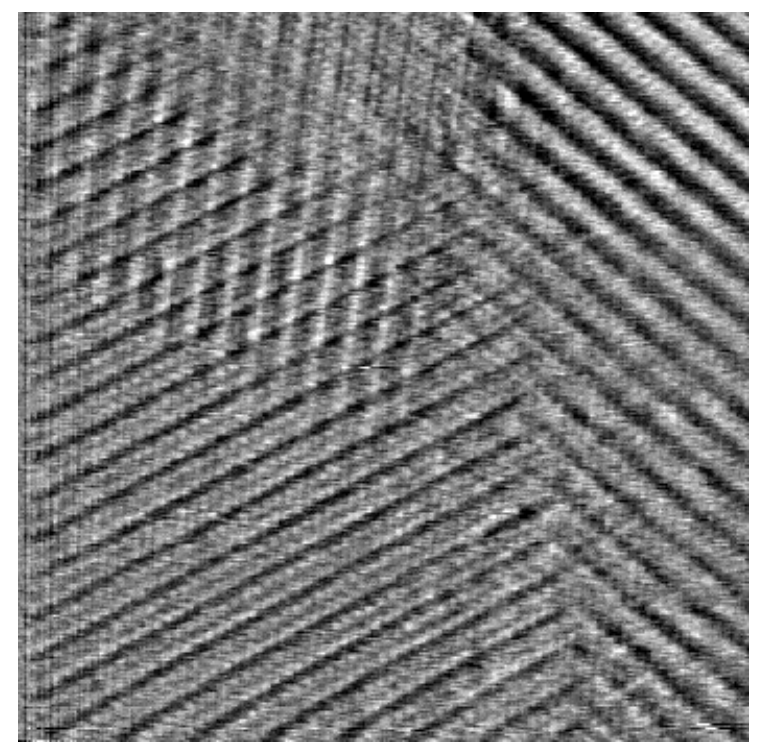

FIG.2. STM image of three domains in $\mathrm{NbTe}_{2}$, separated by strongly modulated boundaries; $(20 \mathrm{~nm})^{2}$ area, constant height mode, $\mathrm{U}_{\mathrm{g}}=0.2 \mathrm{mV}, \mathrm{I}_{\mathrm{t}}=2 \mathrm{nA}$. 